Jurnal Kejuruteraan 30(1) 2018: 1-6

https://doi.org/10.17576/jkukm-2018-30(1)

\title{
An Optimized Structure for Enhancing Optical Absorption of Solar Energy in Elliptical GaAs Nanowire Array Solar Cell
}

\author{
(Pengoptimuman Struktur Bagi Meningkatkan Penyerapan Optik Tenaga Solar dalam GaAs Eliptik Susunan \\ Nanowayar Sel Solar) \\ Nazmus Sakib \\ Electrical and Computer Engineering, University of North Carolina at Charlotte, USA \\ Md. Atiqur Rahman Ahad* \\ "Electrical and Electronic Engineering, University of Dhaka, Bangladesh
}

\begin{abstract}
The enhanced optical absorption of solar energy in a solar cell using nanostructured materials is a very demanding and important research area. Hence, an optimized structure to increase the optical absorption of solar energy in an elliptical GaAs (Gallium Arsenide) nanowire array solar cell was proposed in this paper. The influence of geometric parameters on the optical absorption of elliptical GaAs nanowire solar cells was investigated by exploiting the finite difference in time domain simulations. Based on the design and analysis, the elliptical GaAs nanowire array performed better than any circular GaAs nanowire. It was found that the enhanced performance was due to the reduced reflection from the top surface and a reduced transmission from the bottom surface of the elliptical GaAs nanowire array. The structural parameters of the elliptical GaAs nanowire were optimized by calculating the short circuit current density $\left(J_{\text {sc }}\right)$ for different geometries over the solar spectrum. It was demonstrated that there was an increase of $9.4 \%$ in the $J_{\text {sc }}$ compared with the circular GaAs nanowire under the optimized conditions.
\end{abstract}

Keywords: solar cell; GaAs; nanowire array; solar energy; optical absorption

\section{INTRODUCTION}

If present trend continues, in the year 2025, the global population is likely to reach eight billion and by 2040 it may reach around nine billion (World Population Prospects 2013). As a result, energy consumption is likely to reach in a new height. Most frequently used energy sources for consumption are natural oil, gas and coal. Consequently, quick reductions of some of these limited sources are possible in near future (Das and Islam 2014). To avoid this critical condition and to reduce the dependency on limited energy sources, renewable energy sources become more attractive globally (Selvaraj et al. 2009). Due to advancement in power electronics techniques and decreasing prices, demand of renewable energy sources has grown consistently by $20 \%$ $25 \%$ per annum over the past 20 years (Selvaraj et al. 2009). Among various renewable energy options, solar energy has been an increasing interest for research, mainly due to increasing efficiency of solar cells, technology advancement and economies of scale (Monge et al. 2008). Solar energy offers a clean, abundant, and climate-friendly energy resource to mankind, relatively well-spread over the Earth (Das and Islam 2014). In total, fifteen thousand ExaJoule $\left(1 \mathrm{eJ}=10^{18}\right.$ $\mathrm{J})$ of solar energy are available in the atmosphere per year. And the human consumption is still less than one thousand ExaJoule (Das and Islam 2014, Prinn and Reilly 2014).

In recent years, semiconductor nanowire-based solar cell technologies have been established as a promising alternative to traditional thin-film technologies due to their unique optical and electrical properties (Sandhu et al. 2014). Semiconductor nanowire-based solar cells have higher capabilities to trap incident light which provides more optical absorption and hence increase the overall efficiency of the cells (Kelzenberg et al. 2010, Wang et al. 2014, Lee et al. 2013). Recently, nanowires have become more and more popular in solar cell research due to its large surface to volume ratio, optimized charge separation-collection, intrinsic anti-reflection effect and other excellent properties ( $\mathrm{Wu}$ et al. 2014, Ren et al. 2014). So far, most of the nanowire array-based solar cell technologies are based on the silicon semiconductor (Wen et al. 2011). Regardless of the specific application area, silicon nanowire-based solar cells usually perform better than thin-film solar cells. However, over the last several years, GaAs nanowires have started gaining attention in higherefficiency solar cell research (Wen et al. 2011). Compared to Si nanowires, GaAs nanowires have high absorption coefficient and more proficient in multi-junction applications in order to cover a wider range of solar spectrum for increased efficiency (Wen et al. 2011; Colombo et al. 2009; Tajik et al. 2011; Czaban et al. 2008).

Various recent investigations in terms of simulation and experimental studies have demonstrated that with well-defined diameter and length, NWAs exhibit very high absorption spectra than the thin film with almost similar thickness (Wen et al. 2011; Li et al. 2009; Zhu et al. 2008; Hu and Chen 2007; Garnett and Yang 2010;Kupec et al. 2010;Kelzenberg et al. 
2010; Sakib and Ahad 2015;Gu et al. 2011). Due to optical resonances and diffraction, various nanowire array solar cells can demonstrate inherent light trapping as well as absorption enhancement properties (Ghahfarokhi et al. 2016). Solar cells that are made of semiconductor nanowires have special attention in the research community recently (Yao et al. 2014). It has exclusive optical, structural and electrical properties. NWA depicts the possibility for higher efficiency under diffuse solar light radiance (Ghahfarokhi et al. 2016). Therefore, the exploitation of nanowire array becomes an important research arena for high-efficiency photovoltaics.

Researchers are exploring III-V-based nanowires, especially GaAs to find better optical absorption (Yao et al. 2014; Li et al. 2014;Azizur-Rahman and LaPierre 2015; Ghahfarokhi et al. 2016; Trukhin et al. 2017). Apart from GaAs, few other combinations are explored as well, e.g., InP, GaAs-AlGaAs-GaAs, and InAs nanowire arrays (AzizurRahman and LaPierre 2015; Leahu et al. 2017). However, GaAs-based nanowires are more explored. For example, Z $\mathrm{Li}$ et al. 2014 have done a numerical simulation to propose a GaAs core-shell nanowire array solar cell. On the other hand, Yao et al. 2014 introduced p-i-n-based structure. In order to accomplish increased light absorption efficiency within a suitable structure, it is required to have smart strategies and designs with the correct consideration of the optical properties of the structures. Then we can evaluate the performance of the designed NWA solar cells. GaAs nanowire arrays with axial p-i-n junctions are proposed in (Yao et al. 2014), which has $7.6 \%$ efficiency.

Even though there are studies of optical properties on circular GaAs nanowire array solar cells such as Wen et al. 2011, the works on the similar optical properties on elliptical GaAs nanowire array solar cells are lacking in the literature. Hence, we pondered to an investigation on the optical characteristics of an elliptical GaAs NWA solar cell. In our work, we study and analyze the impact of geometric parameters on the optical absorption of elliptical GaAs nanowire array solar cells. For this purpose, we utilize Finite Difference Time Domain (FDTD) method from Lumerical FDTD solutions (Lumerical Solutions 2017). For this study, we compared the optical absorption of circular GaAs and elliptical GaAs nanowire array solar cells. In particular, we computed the short circuit current density for different geometries of elliptical GaAs NWA solar cell and thus obtained an optimized geometry. The performance of our design of elliptical GaAs NWA solar cell has stronger light absorption in the short wavelength region. It also has broadband light absorption in the long wavelength region. Therefore, we can state that an elliptical GaAs NWA solar cell can demonstrate improved light absorption, compare to a circular GaAs NWA solar cell.

The paper is organized as follows: Section 1 covers the background of the paper. In Section 2, we explain the elliptical nanowire-based solar cells and investigate the optical properties using finite difference time domain method.
Then, we explain the results in Section 3. Finally, we conclude the paper in Section 4.

ELLIPTICAL GAAS NANOWIRE-BASED SOLAR CELL

As mentioned above, the elliptical GaAs nanowire-based solar cell is explored in this paper. We have studied the optical properties of elliptical GaAs NWA-based solar cells. For this analysis, we have exploited the finite difference time domain (FDTD) method (Gu et al. 2011, Yao et al. 2014). In order to compute the energy-dependent transmission $\mathrm{T}(\mathrm{E})$, absorption spectra $\mathrm{A}(\mathrm{E})$, and reflection $\mathrm{R}(\mathrm{E})$, time domain methods are widely used. Before going into the analysis, first, we have presented the design of this solar cell which is shown in Figure 1 (Sakib and Ahad 2015). In this structure, the key parameters are, L (length of the nanowires), $\mathrm{T}$ (thickness of the substrate), P (periodicity), D (major axis length of the ellipse), and d (minor axis length of the ellipse).

In our structure, we select the length of the nanowire as $1 \mu \mathrm{m}$. We also design the thickness of the substrate as $1 \mu \mathrm{m}$. In this case, the periodicity is selected as $0.4 \mu \mathrm{m}$. The main reason for this selection is to provide better light trapping for the solar cell.

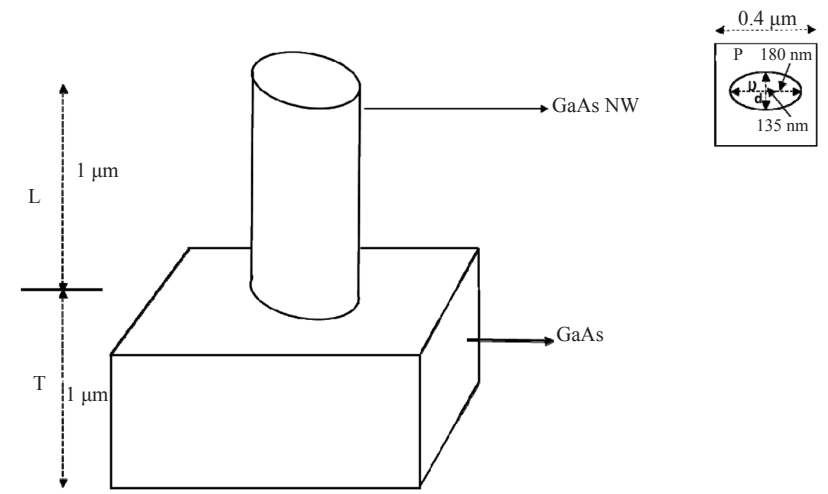

FIGURE 1. Simple design of the GaAs nanowire structure

Figure 2 demonstrates a schematic of a periodic elliptical GaAs nanowire array for our experiment. In order to design a prototype of a periodic square array, we set a few periodic boundary conditions: (i) side boundaries, (ii) perfectly matched layer (PML) boundary condition for the upper boundary, and (iii) the PML for the lower boundary. We simulate the design within this unit cell. The cell eventually represents a periodic square array. The polarization direction of the incident light is parallel to the $\mathrm{X}$-axis, while the propagation direction of the same is parallel to the z-axis (Figure 2). For our study, we investigated the optical properties over the visible wavelength region and the infrared wavelength region of the solar spectrum. Note that the visible region is within $420 \mathrm{~nm}$ to $700 \mathrm{~nm}$, whereas the infrared region is within $700 \mathrm{~nm}$ to $900 \mathrm{~nm}$ of the spectrum. 


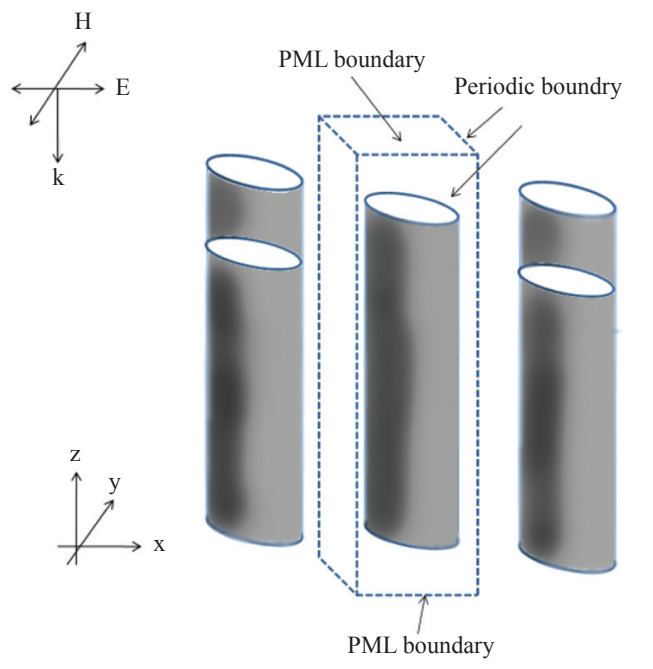

FIGURE 2. A schematic of a periodic elliptical GaAs nanowire array

To determine the optimized geometric configuration of elliptical GaAs NWA over the solar spectrum of interest, short circuit current density $J_{S C}$ is calculated assuming that all photo-generated carriers can contribute to short circuit current using:

$$
J_{s c}=\int_{E_{g}}^{\infty} \frac{A(E) I(E)}{E} d E
$$

where,

$E$ is the photon energy in electron volts $(\mathrm{eV})$,

$A(E)$ is the absorption spectrum of NWA,

$I(E)$ is the solar irradiance spectrum at $\mathrm{AM} 1.5 \mathrm{G}$, and

is the band-gap energy of GaAs.

\section{RESULTS AND DISCUSSION}

First, we investigated the spectral response of circular GaAs NWA with different diameters (D). The diameters selected for this study are $150 \mathrm{~nm}, 180 \mathrm{~nm}, 200 \mathrm{~nm}, 220 \mathrm{~nm}$, and 240nm. In order to compare the performance, the absorbance of a 2 $\mu \mathrm{m} \mathrm{GaAs}$ thin film is also shown. As shown in Figure 3, in short wavelength region $(\lambda-500 \mathrm{~nm})$, compared to GaAs thin film with the same thickness, circular GaAs NWA gives better absorption. The main reason for this enhancement is due to reduced light reflection in the front surface of GaAs NWA. However, in the long wavelength region $(\lambda-550$ $\mathrm{nm})$, we observed a sharp decline in absorption. According to references (Ren et al. 2014, Wen et al. 2011), the main reason for this decline is the sudden reduction in the extinction coefficient of GaAs for these wavelengths. Also, the energy present in the wave plays a significant role in optical absorption. For higher energy waves, more supported modes are available in NWAs which eventually leads to higher absorption by the structure (Wen et al. 2011). We observed a shift of the absorption edges toward long wavelength region as shown in Figure 3. It results in overall broadened absorption band. On the other hand, in the short wavelength region, we observed reduced light absorption. Based on this analysis, it is evident that GaAs NWA with a reduced diameter can attain greater absorption in the long wavelength region.

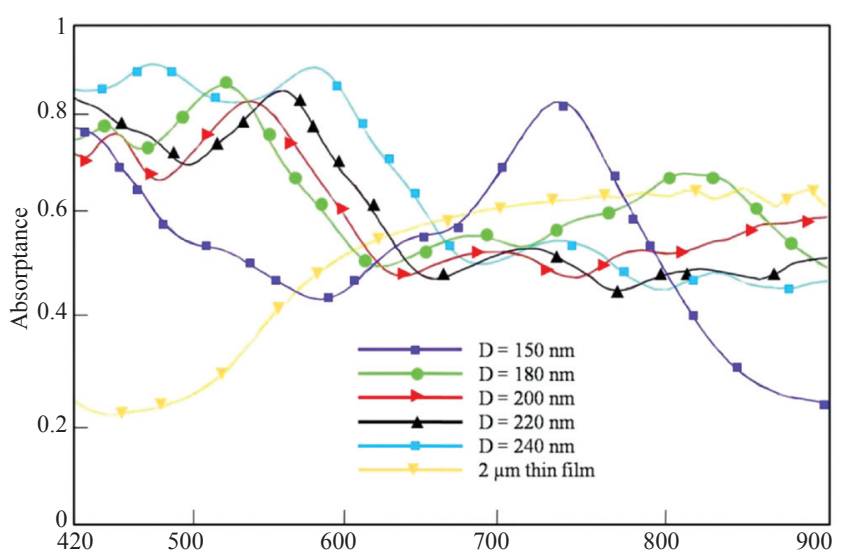

FIGURE 3. Absorbance of a circular GaAs nanowire array with different parameter

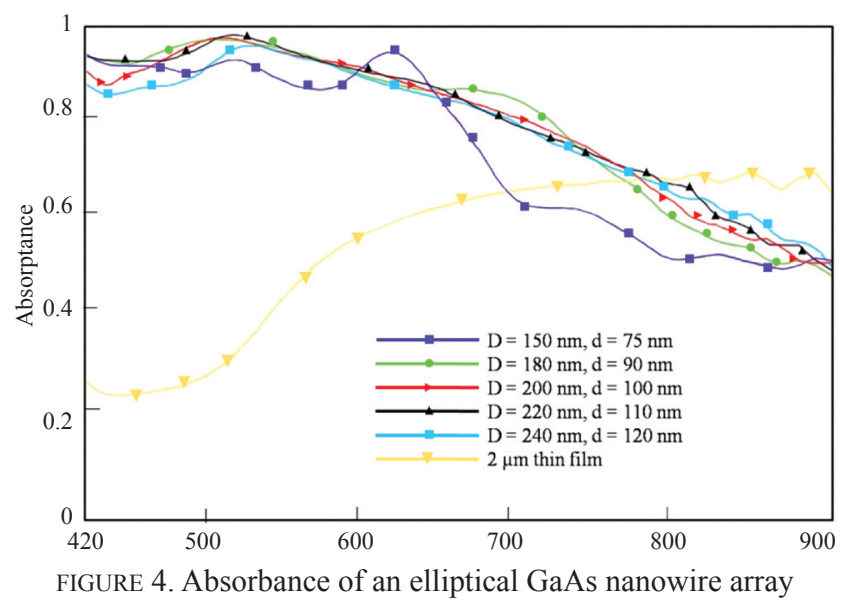

On the other hand, GaAs NWA with a larger diameter can achieve broadband light absorption.

Now, we explore the elliptical design for the GaAs nanowire array. In order to find an optimized elliptical structure, we observed the absorption spectra of NWAs with different major axis length (D) and a fixed aspect ratio of 0.5. As shown in Figure 4, compared with circular GaAs NWAs, elliptical GaAs NWAs maintain overall strong absorption in short wavelength region as well as in the long wavelength region. The main reason for this phenomenon is due to the reduction of reflection and transmission from the top and bottom surfaces. Depending on this finding, it is clear that elliptical GaAs NWAs show the quality of circular NWAs with both short and long wavelength regions.

Furthermore, we investigated the impact of aspect ratio on the optical absorbance of elliptical GaAs NWA. Note that the aspect ratio is defined as the ratio of minor axis to the major axis: $\mathrm{d} / \mathrm{D}$. 


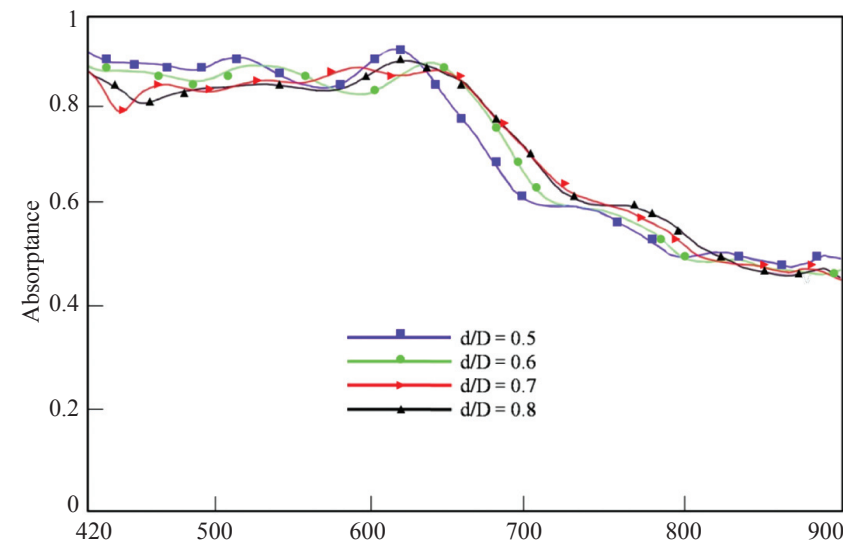

FIGURE 5. Absorbance of elliptical GaAs NWA with major axis length of 150 and different aspect ratios of $0.5,0.6,0.7$, and 0.8

Figure 5 shows the absorption spectra of the elliptical GaAs NWA with different aspect ratio at a fixed diameter of $150 \mathrm{~nm}$. It is evident that the absorption behavior of elliptical GaAs NWA is influenced by its aspect ratio. With a decreasing aspect ratio, enhanced absorption is observed in short wavelength region. Meanwhile, as shown in Figure 5 , absorption in the long wavelength region is suppressed. This result is mainly due to the increase in reflection at the top surface of the NWA.

From the above findings and analysis, it is evident that light absorption of the elliptical GaAs NWA is delicate to the structural variables. By selecting proper major axis length and aspect ratio, absorption of elliptical GaAs NWA can be significantly enhanced. In order to compute the gross light absorption proficiency of an elliptical GaAs NWAs over our desired solar spectrum, we compute the short circuit current density $\left(J_{S C}\right)$ based on the equation 1.

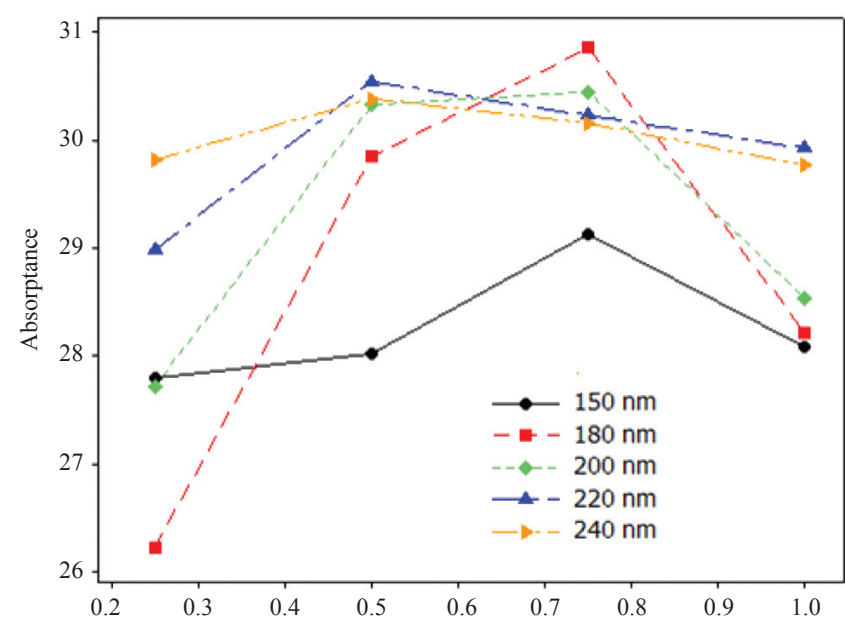

FIGURE 6 . The short circuit current density for different major axis lengths (D) and different aspect ratios (d/D)
Figure 6 depicts $J_{S C}$ for different D as a function of aspect ratio $(\mathrm{d} / \mathrm{D})$. A general increase of $J_{S C}$ is observed when increasing major axis length D from $150 \mathrm{~nm}$ to $220 \mathrm{~nm}$. This trend arises due to the excitation of guided resonance modes (Lee et al. 2013). However, a further increase in D to $240 \mathrm{~nm}$ will lead to a decrease in $J_{S C}$. This trend is attributed to the increase of reflection at the top surface of NWA. From Figure 6 , the maximum $30.86 \mathrm{~mA} / \mathrm{cm}^{2}$ is achieved for major axis length of $180 \mathrm{~nm}$ and $\mathrm{d} / \mathrm{D}$ of 0.75 . However, for a circular array with $\mathrm{D}=\mathrm{d}=180 \mathrm{~nm}$, we observed $J_{S C} 28.21 \mathrm{~mA} / \mathrm{cm}^{2}$. Hence, with optimal diameters of $\mathrm{D}=180 \mathrm{~nm}, \mathrm{~d}=135 \mathrm{~nm}$, $J_{S C}$ is improved by $9.4 \%$.In future, we would like to explore the performance of GaAsnanocone array structure as shown in Figure 7.

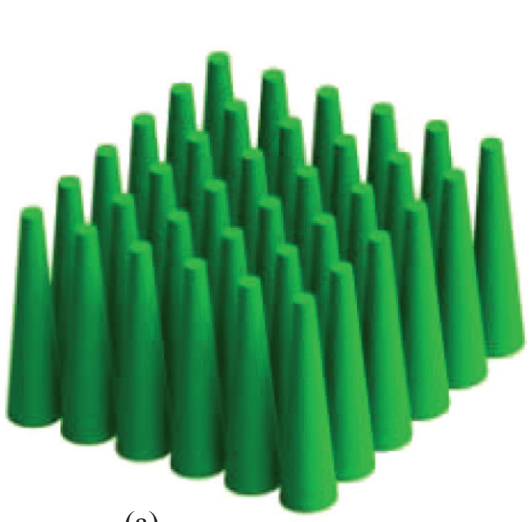

(a)

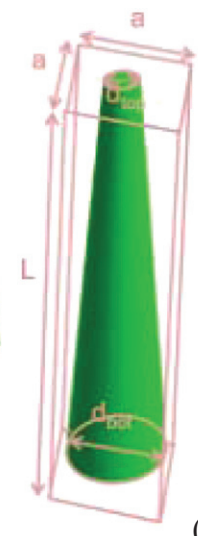

(b)
FIGURE 7. GaAsnanocone array: (a) Graphical representation of a GaAsnanocone array structure. (b) The parameters for the array are length $L$, period $a$, top diameter $d_{\text {top }}$ and bottom diameter $d_{\text {bot }}$

\section{CONCLUSION}

Solar energy is very important to mitigate the future challenges of energy sources. Therefore, extensive research to explore various solar cell arrays that can produce enhanced solar light absorption is necessary. In this paper, by using finite difference time domain (FDTD) method, we investigate the optical characteristics of an elliptical GaAs nanowire array to achieve low-cost but high-efficiency solar cells. We analyze the effect of nanowire diameter (D) and aspect ratio (d/D) on the solar light absorption in elliptical GaAs NWA. According to our study, the elliptical GaAs NWA performs enhanced overall light absorption performance as compared with circular GaAs NWA. We found that the elliptical GaAs NWA can achieve higher short circuit current density $J_{S C}$ of $30.86 \mathrm{~mA} / \mathrm{cm}^{2}$, if we design the major axis length as $180 \mathrm{~nm}$ and the minor axis length as $135 \mathrm{~nm}$. This optimum design parameter for our elliptical GaAs nanowire array can produce $9.4 \%$ more photocurrent as compared with the circular GaAs NWA. This is a significant improvement based on this design. 


\section{ACKNOWLEDGEMENTS}

Authors would like to thank Center for Natural Science and Engineering Research (CNSER) for her support.

\section{REFERENCES}

Azizur, R. \& LaPierre, R. 2015. Wavelength-selective absorptance in GaAs, InP and InAs nanowire arrays. Nanotechnology 26(29): 29520.

Busquets-Monge, S., Rocabert, J., Rodríguez, P., Alepuz, S. \& Bordonau, J., 2008. Multilevel diode-clamped converter for photovoltaic generators with independent voltage control of each solar array. IEEE Transactions on Industrial Electronics 55(7): 2713-2723.

Colombo, C., Hei, M., Gratzel, M. \& Morral, A. F. 2009. Gallium arsenide $\mathrm{p}$-i-n radial structures for photovoltaic applications. Applied Physics Letters 94(17): 173108 1-3.

Czaban, J. A., Thompson, D. A. \& LaPierre, R. R. 2009. GaAs core-shell nanowires for photovoltaic applications. Nano Letters 9(1): 148-154.

Das, N. K. \& Islam, S. M. 2014. Conversion efficiency improvement in gaas solar Cells. In Large Scale Renewable Power Generation, Springer, 53-75.

Garnett, E. \& Yang, P. 2010. Light trapping in silicon nanowire solar cells. Nano Letters 10: 1082-1087.

Ghahfarokhi, O., Anttu, N., Samuelson, L. \& Aberg, I. 2016. Performance of GaAs Nanowire Array Solar Cells for Varying Incidence Angles. IEEE Journal of Photovoltaics 6(6): 1502-1508.

Gu, Z., Prete, P., Lovergine, N. \& Nabet, B. 2011. On optical properties of GaAs and $\mathrm{GaAs} / \mathrm{AlGaAs}$ core-shell periodic nanowire arrays. Journal of Applied Physics 109(6): 064314.

Hu, L. \& Chen, G., 2007. Analysis of optical absorption in silicon nanowire arrays for photovoltaic applications. Nano Letters 7(11): 3249-3252.

Selvaraj, J. \& Rahim, N.A. 2009. Multilevel inverter for gridconnected PV system employing digital PI controller. IEEE Transactions on Industrial Electronics 56(1): 149-158.

Kelzenberg, M. D., Boettcher, S., Petykiewicz, J., TurnerEvans, D., Putnam, M., Warren, E., Spurgeon, J., Briggs, R., Lewis, N. \& Atwater, H. 2010. Enhanced absorption and carrier collection in Si wire arrays for photovoltaic applications. Nature Materials 9(3): 239-244.

Kupec, J., Stoop, R. L. \& Witzigmann, B. 2010. Light absorption and emission in nanowire array solar cells. Optics Express 18(26): 27589-27605.

Leahu, G., Petronijevic, E., Belardini, A., Centini, M., Voti, R., Hakkarainen, T., Koivusalo, E., Guina, M. \& Sibilia, C. 2017. Photo-acoustic spectroscopy revealing resonant absorption of self-assembled GaAs-based nanowires. Scientific Reports, Nature 7: 2833.
Lee, E., Zhou, K., Gwon, M., Jung, J.-Y., Lee, J. \& Kim, D.W. 2013. Beneficial roles of Al back reflectors in optical absorption of Si nanowire array solar cells. Journal of Applied Physics 114(9): 093516-1-093516-6.

Li, J., Yu, H., Wong, S., Zhang, G., Sun, X., Lo, P. G. \& Kwong, D.-L. 2009. Si nanopillar array optimization on Si thin films for solar energy harvesting. Applied Physics Letters 95(11): 033102.

Li, Z., Wenas, Y., Fu, L., Mokkapati, S., Tan, H. \& Jagadish, C., 2014. Numerical simulation on core-shell GaAs nanowire array solar cells. Light, Energy and the Environment, OSA Technical Digest, JW6A.29.

Lumerical Solutions, Inc. 2017. https://www.lumerical.com/ tcad-products/fdtd/ [10 Jan. 2017]

Prinn, R.G. \& Reilly, J.M. 2014. Energy and Climate Outlook.MIT Joint Program on the Science and Policy of Global Change. http://globalchange.mit.edu/research/ publications/other[1 June 2017]

Ren, R., Guo, Y. X. \& Zhu, R. H. 2014. Enhanced absorption in elliptical silicon nanowire arrays for solar energy harvesting. Optical Engineering 53(2): 027102.

Sakib, N. \& Ahad, M.A.R. 2015. Enhanced absorption of solar energy in elliptical GaAs nanowire array solar cells. In Informatics, Electronics \& Vision (ICIEV), $4^{\text {th }}$ International Conference on IEEE.

Sandhu, S., Yu, Z. \& Fan, S. 2014. Detailed Balance Analysis and Enhancement of Open-Circuit Voltage in SingleNanowire Solar Cells. Nano Letters 14(2): 1011-1015.

Tajik, N., Peng, Z., Kuyanov, P. \& LaPierre, R. R. 2011. Sulfur passivation and contact methods for GaAs nanowire solar cells. Nanotechnology 22(22): 225402.

Trukhin, V., Bouravleuv, A., Mustafin, I., Kakko, J. \& Lipsanen, H. 2017. Optically excited $\mathrm{THz}$ generation from ordered arrays of GaAs nanowires. Procedia Engineering 201: 100-104.

Wang, B., Stevens, E. \& Leu, P. W. 2014. Strong broadband absorption in GaAsnanocone and nanowire arrays for solar cells. Optics Express 22: A386-A395.

Wen, L., Zhao, Z., Li, X., Shen., Y., Guo, H. \& Wang, Y. 2011. Theoretical analysis and modeling of light trapping in high efficiency GaAs nanowire array solar cells. Applied Physics Letters 99: 143116(1-3).

United Nations Department of Economic and Social Affairs/ Population Division. 2013. World Population Prospects: The 2012 Revision, Key Findings and Advance Tables. Working Paper No. ESA/P/WP.227, United Nations, New York.http://esa.un.org/wpp/ 1-50

Wu, L., Li, S., He, W., Teng, D., Wang, K. \& Ye, C. 2014. Automatic Release of Silicon Nanowire Arrays with a High Integrity for Flexible Electronic Devices. Scientific Reports, Nature 4: 3940(1-7).

Yao, M., Huang, N., Cong, S., Chi, C.-Y., Seyedi, M. A., Lin, Y.-T., Cao, Y., Povinelli, M., Dapkus, P. \& Zhou, C. 2014. GaAs Nanowire Array Solar Cells with Axial p-i-n Junctions. Nano Letters 14(6): 3293-3303. 
Zhu, J., Yu, Z., Burkhard, G., Hsu, C., Connor, S., Xu, Y., Wang, Q., McGehee, M., Fan, S. \& Cui, Y. 2009. Optical absorption enhancement in amorphous silicon nanowire and nanocone arrays. Nano Letters 9(1): 279-282.

\section{Nazmus Sakib}

Electrical and Computer Engineering,

University of North Carolina at Charlotte,

9201 University City Blvd, Charlotte, NC 28223, USA

Md. Atiqur Rahman Ahad*

Dept. of Electrical and Electronic Engineering, University of Dhaka, Dhaka 1000, Bangladesh

*Corresponding Author; email: atiqahad@du.ac.bd

Received date $: 9^{\text {th }}$ October 2017

Accepted date: $20^{\text {th }}$ February 2018

In Press date: $1^{\text {st }}$ April 2018

Published date : $30^{\text {th }}$ April 2018 